\title{
XXV. Nachtrag zu den „krystallographischen Studien am Eisenglanz und Titaneisen vom Binnenthal".
}

\author{
(Diese Zeitschr. 1, ว̈62 u. f., Taf. XXIV.) \\ Von Demselben. \\ (Hierzu Taf. XV. Fig. 18-20.)
}

Durch die Untersuchung einer grösseren Anzahl von Eisenglanzkrystallen von der Alp Lercheltini im Binnenthal, die mir theils wiederum von Herrn G. Seligmann in Goblenz, theils von Herrn Dr. G. H in tze dahier mit der grössten Zuvorkommenheit zur Bearbeitung überlassen wurden, bin ich im Stande, meine fruhere Beschreibung des dortigen Vorkommens durch neue Beobachtungen zu ergänzen.

Die untersuchten Krystalle gehören zum grössten Theil den bereits beschriebenen Typen an, am häufigsten dem ersten; vierten und siebenten. Meine fruhere Angabe, nach der der vierte Typus sich seltener finden sollte, ist demnach zu berichtigen. Auch wurden noch vier neue von den beschriebenen verschiedene Ausbildungsweisen beobachtet, die unten ausführlicher betrachtet werden sollen.

Die dem ersten Typus zugehörigen Krystalle waren weitaus in den meisten Fällen so entwickelt; wie früher angegeben wurde. Nur wenige Krystalle besassen ein etwas abweichendes Aussehen. Die einen, 7-10 mm breit und $2-3^{\text {mm }}$ dick, gehören wegen des Vorwaltens der Basis zu den schon früher besprochenen tafelförmig ausgebildeten Krystallen. Während aber sonst an diesen das primäre Rhomboëder $r=R(10 T 1)$ nur klein ist oder ganz fehlt, ist es hier auf Koslen des Skalenoëders $A=-\frac{1}{2} R_{3}$ $x$ (10. 20. $\overline{3} \overline{0} .27$ ) sehr gross entwickelt; dabei besitzt es nicht, wie gewöhnlich, matte Flächen, sondern ist ganz eben und spiegelnd. Das Skalenoèder $B={ }_{7}^{20} R \frac{8}{5} x(26.6 . \overline{3} \overline{2} .7)$ mit gerundeten, aber stark glänzenden Flächen waltet gegenuber $A$ vor (vgl. Fig. 1 auf Taf. XXIV des ersten Bandes). 
Grössere Abweichungen von der typischen Ausbildung zeigle ein anderer Krystall, welcher sich in der Sammlung des Herrn G. Seligman befindet; erst durch Messung mehrerer Winkel wurde seine Zugehı̈rigkeit zum ersten Typus ausser Frage gestellt. Er ist etwa $17^{\mathrm{mm}}$ breit und $2 \frac{1}{2} \mathrm{~mm}$ dick, ebenfalls durch Vorwalten der Basis tafelförmig. Ausser der letzteren sind nur noch die beiden Skalenoëder $A$ und $B$ zur Ausbildung gelangt. Von diesen herrscltt das negative $B$ beträchtlich vor; $A$ tritl nur auf als Abstumpfung der Ecken, welche je eine obere und eine untere Flïche des steilen Skalenoëders $B$ mit der Basis bilden. Sämmtliche Flächen sind glänzend. Die Basis $c$ ist nur am Rande eben und parallel der Combinationskante von $c$ mit $A$ fein gestreift; in der Mitte befindet sich eine Anhäufung rosettenförmig gruppirter Eisenglanzblättchen, die nur wenig gegen die Ebene der Basis geneigt sind. Das Skalenoëder $A$ ist ziemlich eben; dagegen ist $B$ stark gekrümmt und durch öfteres Alterniren der beiden die stumpfe Polkante bildenden Flächen wellig gebogen. Die Messung ergab für den Winkel von $A$ zur Basis den Werth $56^{\circ} 56^{\prime}$ (ber. $56^{\circ} 59^{\prime}$ ), für den stumpfen Polkantenwinkel $31^{\circ} 52^{\prime}$ (ber. $3 t^{\circ} 50^{\prime}$ ). Bei $B$ liess sich wegen der starken Wölbung in horizontaler Richtung nur der Winkel zur Basis, auch dieser nur ganz approximativ, bestimmen, er war ca. $83^{\circ}$ (ber. $\left.81^{0} 24^{\prime}\right)$.

Grosse Aehnlichkeit mit Typus 2 haben zwei auf einer Stufe befindliche, pyramidal ausgebildete Krystalle, von denen der grössere mit einer Seite frei aus dem Gneiss hervorragt. Er ist $5^{\mathrm{mm}}$ breit und $3^{\mathrm{mm}}$ dick. Die vorherrschenden Formen sind die Basis und ein Skalenoëder, welches den Messungen zufolge als $G=-\frac{7}{19} R \frac{23}{7}=x(8.15 . \overline{2} \overline{3} .19)$ betrachtet werden kann; untergeordnet treten auf ein Rhomboëder, welches die stumpfe Polkante von $G$ anscheinend gerade abslumpft, ein Skalenoëder, welches die spitze Polkante zuschärft, demnach eine ähnliche Lage wie $D=$ ${ }_{2}^{7}{ }^{7} R{ }_{7}^{23}=x(15.8 . \overline{2} \overline{3} .22)$ besitzt (vgl. d. Zeitschrift I, Taf. XXIV, Fig. 2), und eine nur ganz matte, undeutliche Fläche, welche zwischen den beiden Skalenoëdern liegl und anscheinend der Pyramide zweiter Ordnung $Q=$ $\frac{6}{5} P \geqslant(33 \overline{6} 5)$ entspricht. Sämmtliche Flächen mit Ausuahme der Basis, des Rhomboëders und zweier Flächen von $G$ sind matt; es konnten deshalb nur weuige Winkel gemessen werden. Die Basis ist wie an dem früher beschriebenen Krystall nach der Mitte hin durch kleine Unebenheiten rauh. Das Skalenoëder $G$ ist stark gestreift parallel der Combinationskante mit dem Rhomboëder; der stumpfe Polkantenwinkel war $33^{0} 54^{\prime}$ (ber. 3 ł $^{0} 8^{\prime}$ ), der Winkel zur Basis 58" $56^{\prime}$ (ber. $59^{\circ} 7^{\prime}$ ). Von dem Rhomboëder liess sich wegen der undeutlichen breilen Reflexe, welche die Flächen des Skalenoëders $G$ geben, nicht mit Sicherheit entscheiden, ob es in der That die stumpfe Polkante von $G$ gerade abstumpft, wie es das primäre negative Rhomboëder thun würde. Es scheint vielmehr, da der Winkel, welchen die Groth, Zeitschrift f. Krystallogr. II. 
glänzende Rhomboëderfläche mit der Basis bildet, nur $56^{\circ} 23^{\prime}$ beträgl und dieser Werth wegen der verbältnissmässig guten Reflexe nur wenige Minuten von dem wahren Werthe abweichen kann, slatt $-R\langle 01 T 1\rangle$, dessen Winkel zur Basis gleich $57^{0} 31^{\prime}$, ein flacheres Rhomboëder, etwa $-\frac{2}{2} \frac{3}{4} R$ $=(0.24 . \overline{2} \overline{4} .23)$, dessen Winkel zur Basis sich auf $56^{\circ} 24^{\prime}$ berechnet, vorzuliegen. Die anderen Flächen, welche als $D$ und $Q$ gedeulet werden könnten, waren zu matt und uneben; als dass uber ihr Zeichen entscheidende Winkelmessungen hätten vorgenommen werden können; die als $Q$ aufzufassende Fläche schien mit den benachbarten Flächen von $G$ und dem Rhomboëder in eine Zone zu fallen, wie dies auch annähernd der Fall sein müsste.

An einigen kleinen Krystallen des dritten Typus, auf welche im Allgemeinen die früher gegebene Beschreibung passt, wurden zuweilen noch kleine dreieckige, sehr glänzende Flächen beobachtet, welche die Ecken abstumpfen, die von den Flächen $E$ oben, $E$ unten und $P$ gebildet werden (also z. B. die Ecke (11. 7. $\overline{1} \overline{8} .22,7.11 . \overline{1} \overline{8} . \overline{2} \overline{2}, 24 \overline{6} 7$ ); vgl. Fig. 3 der cit. Arbeit). Aus den Messungen ergibt sich mit voller Bestimmtheit, dass diese Flächen der Pyramide zweiler Ordnung $n=\frac{4}{3} P 2$ (22743) angehören. Sie sind stels eben und spiegelnd, aber immer nur sehr klein, sodass sie nicht mehr in horizontalen Mittelkanten zum Durchschnitt kommen.

Ein Krystall des vierten Typus zeigle das Prisma $b=\infty R(10 T 0)$ ziemlich gross. An einem andern, etwa $3 \frac{1}{2}^{\mathrm{mm}}$ breiten und $\frac{1}{2}{ }^{\mathrm{mm}}$ dicken Krystall war nächst der Basis das Skalenoëder $F=\frac{4}{13} R \frac{7}{2}=\varkappa(9 . \ddot{b} . \overline{1} \bar{l} .13)$ die vorwaltende Fläche. $F$ war glatt und spiegelnd. Ausserdem war noch das Rhomboëder $r=R(10 \overline{1} 1)$ vorhanden, aber verhältnissmässig nur klein. Die ubrigen Formen treten nur ganz untergeordnet auf. Eine äusserst schmale, etwas gerundete Fläche stumpft die Mittelkante von $F$ gerade ab (Winkel gegen $F$ beob. ca. $35^{0}$, ber. $35^{0} 5^{\prime}$ ) und besitzt daher das Zeichen $\infty R \frac{7}{2}=(9.5 . \overline{1} \overline{4} .0)$. Das Prisma $\infty R \frac{7}{2}$ ist noch nicht am Eisenglanze bekannt.

Die neu beobachteten Typen sind folgende:

\section{Typus.}

Ein auf Gneiss aufgewachsener Krystall, $1: 3^{\mathrm{mm}}$ lang und $2^{\mathrm{mm}}$ dick, und ein loser Krystall, $25^{\mathrm{mm}}$ lang, $15^{\mathrm{mm}}$ breit und $4^{\mathrm{mm}}$ dick, besitzen beide die Combination der Flächen $c=o R(0001) r=R\left(10 T_{1}\right), e=-\frac{1}{2} R(01 \bar{T} 2)$, $v=-\frac{9}{2} R(09 \overline{9} 2)$ mit zwei schmalen negativen Rhomboëdern, der Pyramide zweiler Ordnung $n=\frac{4}{3} P_{2}(2243)$ und den Skalenoëdern $F=$ $\frac{4}{13} R \frac{7}{2}=\varkappa(9.5 . \overline{4} .13), M=-\frac{7}{18} R 3=x(7.14 . \overline{2} \bar{T} .18)$ und $\Sigma=$ $-\frac{7}{2} R \frac{8}{7}=x(1.15 . \overline{1} \overline{6} .4)$; (vgl. Fig. 18, in welcher alle Flächen mit Aus- 
nahme der beiden schmalen negativen Rhomboëder gezeichnet sind). Durch Vorwalten der Basis sind die Krystalle talelförmig. Von den übrigen Formen ist an dem losen Krystall das Skalenoëder $F$ bei weitem am grössten entwickelt; an dem aufgewachsenen sind die Flächen der Rhomboëderzone die vorherrschenden, wodurch derselbe, von der Basis betrachtet, einen sechsseitigen Umriss erhält. In ihrer Ausbildung zeigen die Krystalle eine gewisse Aehnlichkeit mit denen des vierten Typus, mit welchem sie auch die Skalenoëder $\boldsymbol{F}$ und $\boldsymbol{M}$ und das Rhomboëder $\boldsymbol{r}$. gemein haben.

Die Basis ist nur am Rande glänzend und glatt oder parallel den Rhomboëderkanten feingestreift, nach der Mitle hin jedoch sehr uneben durch kleine dem Hauplindividuum nahezu parallel gestellte und rosettenförmig gruppirte dunne Eisenglanzkryställchen und durch ein- und aufgewachsene Rutilnadeln in der hekannten regelmässigen Anordnung.

In der Rhomboederzone ist $r$ am grössten entwickelt; klein, aber eben und glänzend ist $\nu$; etwas matt und sehr gerundet $e$. Zwischen $e$ und $\nu$ liegen, ganz schmal ausgebildet, das Rhomboëder $-\frac{5}{9} R=(05 \overline{5} 9)$ und ein zweites, welchem nach der allerdings nur approximativen Messung das Zeichen $-\frac{8}{11} R==(0.8 . \overline{8} .11)$ zukommen wurde. Die beobachteten und berechneten Winkel zur Basis sind folgende:

$$
\begin{aligned}
& \text { fur } e \text { beob.: } 39^{\circ} 13^{\prime}, 36^{0} 56^{\prime} \text {, ber.: } 38^{0} 7^{\prime} \text {, } \\
& \text { für - } \frac{5}{9} R \text { beob. : } 40^{0} 33 \frac{1}{2}^{\prime}, 40^{\circ} 35 \frac{1^{\prime}}{2}, 41^{0} 33 \frac{1}{2}^{\prime}, 41^{0} 38^{\prime} \text { ber.: } 41^{0} 6^{\prime} \text {, } \\
& \text { für }-\frac{8}{11} R \text { beob. : } 48^{0} 42^{\prime} \text {, } \quad \text { ber. : } 48^{\circ} 48^{\prime} \text {, } \\
& \text { für } \quad \nu \text { beob.: } 81^{0} 34^{\prime}, 81^{0} 37 \frac{1}{2}^{\prime}, 82^{0} 10^{\prime} \quad \text { ber.: } 81^{\circ} 57^{\prime} \text {. }
\end{aligned}
$$

Die Pyramide $n=\frac{4}{3} P 2$ ist eben und glinzend, aber immer sehr klein, sodass die entsprechenden oberen und unteren Flächen nicht mehr in der Mittelkante zum Durchschnitt kommen.

Von den Skalenoëdern ist $F=\frac{4}{13} R \frac{7}{2}$ am grösslen; seine Flächen sind sebr oft etwas gerundet und die Messungen daher nur approximative; doch folgt aus denselben, dass es mit dem schon an Krystallen des vierlen Typus beohachteten Skalenoeder $F$ identisch ist. Die negativen Skalenoëder sind beide von gleicher Grösse, aber kleiner als $F$ und besitzen ebenfalls zum Theil gerundete Flächen. Den gemessenen Winkeln zufolge ist das flache als $M=-\frac{7}{18} R 3$, das steile $\Sigma^{\prime}$ als ein Skalenoëder mit dem Zeichen $-\frac{4 P \frac{16}{15}}{2}=-\frac{7}{2} R \frac{8}{7}=\varkappa(1.15 . \overline{1} \overline{6} .4)$ zu deuten. Fur letzteres sind die beobachteten Winkel *) $U=81^{0} 20 \frac{1}{2}, X=6^{0} 56 \frac{1}{2}^{\prime}$, die berechneten Werthe $U=80^{\circ} 44^{\prime}, X=6^{0} 20^{\prime}$.

*) Es bedeutet auch hier $U$ den Winkel zur Basis, $Y$ den Winkel der stumpfen, $X$ den der scharfen Polkante und $Z$ den Mittelkantenwinkel. 


\section{Typus.}

Ein $14^{\mathrm{mm}}$ breiter und $21^{\mathrm{mm}}$ dicker Krystall, aufgewachsen auf Gneiss, besitzt die Formen $c=o R, r=R(1011)$ und $n=\frac{4}{3} P 2(22 \pi 3)$ in Combination mil $u=\frac{1}{4} R(10 \overline{4} 4)$, einem matten Prisma (?) $b=\infty R(10 \overline{4} 0)$, dem Skalenoëder $A=-\frac{1}{3} R \frac{7}{2}=-\frac{\frac{6}{7} P \frac{14}{9}}{2}=x(5.9 . \overline{1} \overline{4} .20)$, einem positiven Skalenoëder $S$, welches anscheinend mit $n$ und $r$ in einer Zone liegt, und mehreren sehr schmalen und ganz gekrümmten Flächen, die als Abrundung der Kanten einiger der genannten Flächen mit der Basis aufzutreten pflegen. Durch Vorwalten der Basis ist er flach tafelförmig; dabei besitzt er in Folge grösserer Ausbildung des Rhomboëders $r$ gegenüber den andern Flächen, von oben betrachtet, einen gerundet dreiseitigen Umriss (Fig. 19). Der ganze Krystall besteht aus mehreren nicht vollkommen parallel orientirten Theilen; die Messungen waren deshalb zum Theil nur ganz approximative.

Die Basis $c$ ist im Ganzen glatt und glänzend, nur ist sie durch mehrfache nicht parallele An - und Auflagerungen einzelner Theile des Krystalls, sowie durch ein- und aufgewachsene Rutilnadeln uneben und höckerig.

Das Rhomboëder $r=R$ ist unter den Randflächen am grössten entwickelt; es ist vollkommen eben und gibt bei der Messung sehr scharfe Reflexe. Als ganz schmale Abstumpfung der Kante von $r$ mit $c$ wurde $u=\frac{1}{4} R(10 \overline{1} 4)$ beobachtet; der gemessene Winkel zur Basis betrug $20^{\circ} 54^{\prime}$, der berechnete $210^{\circ} 26^{\prime}$. Eine etwas matte Fläche, welche anscheinend in die Rhomboëderzoné fällt, könnte als $b={ }^{\prime} \infty(10 \bar{T} 0)$ gedeutet werden, oder auch, da sie in horizontaler Richtung etwas gerundet ist, als ein dihexagonales Prisma mit einem stumpfen Winkel, der nur wenig von $180^{\circ}$ abweicht.

Die Pyramide 2. Ordnung $n$ ist als kleine, aber ebene und stark glänzende Fläche vorhanden. Zwischen ihr und dem Rhomboëder $r$, anscheinend in der Zone mit beiden Flächen, liegt ein positives Skalenoëder $S$ von matter granulirter Beschaffenheit. Da dasselbe keinen Reflex gibt, konnte seine Neigung zum Rhomboëder $r$ nur annähernd bestimmt werden, sie betrug $24011^{\prime}$. Hieraus würde sich für $S$ das Zeichen $\frac{1}{13} R 17=\frac{\frac{17}{13} P \frac{17}{9}}{2}$. $=x(9.8 . \overline{7} \overline{7} .13)$ ergeben, für welches der Winkel zu $R$ sich zu $24012^{\prime}$ berechnet. Doch weichen die Messungen des Winkels zur Basis und des spitzen Polkantenwinkels ca. $5^{0}$ von den berechneten Werthen ab, was zum Theil wohl auch seinen Grund in der Zusammensetzung des Krystalls aus mehreren nicht parallel orientirten Theilen haben mag; es kann daher der beobachteten Fläche $S$ das angeführte Zeichen nicht mit Bestimmtheit gegeben werden. Bessere Messungen gestattete das kleine negative Skalenoëder $\boldsymbol{A}$, welches als schmale Abstumpfung der Ecken zwischen $n, c$ und der als $b$ 
gedeuteten Flïche auftritt. Der Winkel zur Basis betrug $43^{0} 54^{\prime}$, der slumpfe Polkantenwinkel $28^{\circ} 30^{\prime} ;$ die für $-\frac{1}{5} R \frac{7}{2}=-\frac{\frac{7}{10} P \frac{14}{9}}{2}=$

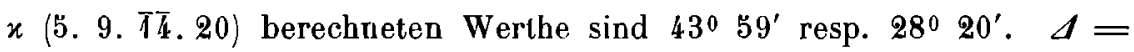
$-\frac{1}{8} R \frac{7}{2}$ ist neu für den Eisenglanz.

\section{Typus.}

Hierher zu rechnen sind mehrere Krystalle, die mit solchen des 7. Typus zusammen vorkommen, aufgewachsen auf Gneiss, etwa $8^{\mathrm{mm}}$ breil und $\frac{3}{4}^{\mathrm{mm}}$ dick; und ein grösserer Krystall, $17^{\mathrm{mm}}$ breit und $1 \frac{1}{2} \mathrm{~mm}$ dick, auf einer andern Stufe, zusammen mit einem oberflächlich braungelben Mineral, dessen Inneres in Farbe und Beschaffenheit des Bruchs an Magneteisen erinnert, während seine Krystallform als Combination von Oktaëder und Hexaëder gedeutet werden könnte. Die Eisenglanzkrystalle sind sämmtlich Zwillinge nach der Basis. Namentlich flächenreich ist ihre Rhomboëderzone. Es wurden die Formen $c=o R(0001), \varphi=\frac{5}{8} R(50 \overline{5} 8), \Pi=\frac{5}{4} R$ (5054), $r=R\left(10 T_{1}\right), \Theta=2 R(20 \overline{2} 1), e=-\frac{1}{2} R(01 \bar{T} 2), N=-\frac{5}{1} R$ (0554), $s=-2 R(02 \overline{2} 1), n=\frac{3}{3} \boldsymbol{2}(\mathbf{2} 2 \overline{4} 3)$ beobachtet in Combination mit vier nicht näher bestimmbaren Skalenoëdern $\Phi, \Lambda, \Omega$ und $R$ (vgl. Fig. 20, in welcher die Combinationskanten der übrigen Flächen mit diesen Skalenoëdern von unbekannten Zeichen nur annähernd richtig gezeichnet werden konnten). Nach der Basis sind die Krystalle flach tafelartig; die Zwillingsgrenze verläuft in der Regel sehr unregelmässig.

Die Basis ist im Allgemeinen sehr eben und glatt, nur zuweilen am Rande feingestreift parallel den Combinationskanten mit den Rhomboëdern.

Unter den Seitenflächen zeichnen sich die Rhomboëder durch Glanz und ebene Beschaffenheit aus; sie sind meist nur ganz schmal entwickelt, oft sind auch einige Flächen etwas breiter als die übrigen ausgebildet. Die beobachteten und berechneten Werthe der Winkel zur Basis sind folgende :

$$
\begin{aligned}
& \begin{array}{crr}
\text { für } \varphi=(50 \overline{5} 8) & \text { für } r=(10 \bar{T}) & \text { für } I I=(50 \overline{5} 4) \\
\text { beob.: } 44^{0} 0^{\prime} & \text { beob.: } 57014^{\prime} & \text { beob.: } 63^{0} 25^{\prime}
\end{array} \\
& 44^{\circ} \mathrm{J6}^{\prime} \quad \quad 57^{\circ} 22^{\prime} \quad 62^{\circ} 41^{\prime} \\
& 63017^{\prime} \\
& \text { ber.: } 44^{0} 28^{\prime} \text { ber, : } 57031^{\prime} \text { ber.: } 63^{\circ} 0^{\prime}
\end{aligned}
$$

\begin{tabular}{|c|c|c|c|}
\hline lür $\Theta=(20 \overline{2} 1)$ & für $e=(01 \bar{\top} 2)$ & für $N=(05 \overline{5} 4)$ & $\mathrm{fuủ} \mathbf{r} \cdot s=(02 \overline{\mathbf{z}} \downarrow)$ \\
\hline beob. : $73^{0} 12^{\prime}$ & beob. : $39^{0} 40^{\prime}$ & beob.: $62^{\circ} 57^{\prime}$ & beob.: $72^{0} 19^{\prime}$ \\
\hline $72^{\circ} 56^{\prime}$ & $37^{\circ} 21^{\prime}$ & $63^{0} 16^{\prime}$ & $72^{\circ} 38^{\prime}$ \\
\hline $7.2020^{\prime}$ & ber.: $38^{\circ} 38^{\prime}$ & ber.: $63^{\circ} 0^{\prime}$ & ber.: $72020^{\prime}$. \\
\hline
\end{tabular}

Die Pyramide 2. Ordnung $n$ ist immer spiegelnd, aber nur sebr klein. Ebenfalls sehr schmal und etwas gerundet ist das Prisma $a=\infty P \mathscr{2}(1 / \overline{\mathcal{Q}} 0)$; (in der Figur ist dasselbe nicht gezeichnet worden). 
Die Skalenoëder zeigen sehr stark gekrümmte Flächen; eine Bestimmung derselben war nicht möglich. Am wenigsten gerundel sind die flachen Skalenoëder $\Phi$ und $\Lambda$, von denen das erstere ein positives, das zweite ein negativès ist. Sie schneiden die Axen nicht in demselben Verhältnisse, wie es den Anschein hat, denn ihre gegenseitigen Combinationskanten convergiren auf der Seite der negativen Rhomboeder nach oben. Die Flächen beider Skalenoëder liegen aber scheinbar mit der Pyramide 2. Ordnung $n$ und den steilen Skalenoëdern $\Omega$ resp. $R$ in einer Zone, in Folge dessen die Flächen von $n$ in der Regel die Gestalt eines Rhombus haben. Für $\boldsymbol{\Phi}$ wurde der Winkel zur Basis zu $47^{\circ} 50^{\prime}$, für $\Lambda$ zu 48038' bestimmt; der Winkel an der Kante $(\Phi, \Lambda)$ war annähernd $16^{0} 33^{\prime}$. Für die steilen Skalenoëderflächen lässt sich, da die Zwillingsgrenze zu unregelmässig verläuft uñ nicht allenthalben deutlich erkannt werden kann, nicht mit Sicherheit entscheiden, ob sie nur einem Skalenoëder angehören, welches dann ein negalives $(\Omega)$ wäre, oder ob sie zum Theil auch dem positiven Skalenoëder mit demselben Zeichen $(R)$ entsprechen. Letzlere Annahme scheint mir die wahrscheinlichere. Die mit $\Omega$ und $R$ bezeichneten Flächen sind sämmtlich stark gestreift in der Weise, dass die Streifen auf je zwei zugehörigen, den stumpfen Polkantenwinkel einschliessenden Flächen nach oben divergiren. Der stumpfe Polkantenwinkel kann etwa $12^{\circ}$ betragen.

\section{Typus.}

Durch die Güle des Herrn Dr. Hin lze dahier, erhielt ich eine Gruppe dick tafelartiger Krystalle zur Untersuchung, von denen der grösste etwa $20^{\mathrm{mm}}$ breit und $5^{\mathrm{mm}}$ dick ist. Die vorherrschenden Formen sind $c=o R(0001), a=\infty P 2(11 \overline{2} 0)$ und $b=\infty R(10 T 0) ; a$ und $b$ sind etwa von gleicher Grösse. Die einzelnen Krystallindividuen, meist nur wenig gegeneinander geneigt, sind fächerförmig angeordnet; nur wenige sind quer gegen die übrigen gestellt. Die Flächen sind sehr uneben und zum Theil bedeckl von feinen Chloritschüppchen. Die Basis ist ausserdem noch rauh durch aufgewạchsene kleine Eisenglanzblattchen und vereinzelle Rutilnadeln in der regelmässigen Stellung.

An einem Krystall wurde auch eine Fläche der Pyramide 9. Ordnung $n=\frac{4}{3} P 2$ (22 43 ) heobachtet. Die Combinationskanten von $c$ mit dieser Fläche und mit $\infty R$ waren abgerundet durch zwei nicht sicher zu bestimmende Flächen, die elwa als $\varkappa=\frac{1}{3} P \geq(1,1 . \overline{2} .10)$ und $\pm \frac{1}{4} R$ gedeutet werden könnten. Approximative Messungen ergaben für die Winkel der sehr gekrümmten Flächen zur Basis die Werthe $16^{0} 54 \frac{1}{2}^{\prime}$ resp. $20^{\circ} 53^{\prime}$; die berechneten sind $10^{0} 13^{\prime}$ resp. $21026^{\prime}$.

Eine Stufe aus der Sammlung des Herrn Selig man n in Coblenz zeigt auf einem sehr quarzreichen Gneisse neben Krystallen von Quarz, Adular und Glimmer einen Eisenglanzkrystall von etwa $13^{\mathrm{mm}}$ Breite. Derselbe be- 
steht aus einer grösseren Anzahl (etwa 20 ) einzelnen Individuen, die in der obenerwähnten Weise fächerförmig gruppirt sind derart, dass der Krystall an der einen Seite eine Dicke von circa $6^{\mathrm{mm}}$, an der andern von $11^{\mathrm{mm}}$ besitzt. Die einzelnen flach tafelförmigen Individuen sind begrenzt durch dic Flächen $c=o R(0001), a=\infty P 2$ (11/20) und $b=\infty R(10 \overline{1} 0)$. Von den Prismen ist nur das erstere gross ausgebildet. Das schmale Prisma muss als $b=\infty R$ gedeutet werden, weil an einem Individuum eine kleine Fläche, welche die Kante zwischen diesem Prisma und der Basis abslumpft, der Messung zufolge das Rhomboëder $r=R(10 T 1)$ ist. Die Basis ist nur.am Rande spiegelnd und eben, in der Mitte sehr rauh und uneben. Die Flächen des Prismas $a$ sind zwar theilweise glatt, aber gewöhnlich in vertikaler Richtung stark gerundet; $b$ ist verhältnissmässig sehr eben.

Bei Betrachtung dieser letzten Typen wurde, wie auch in den fruheren Arbeilen über die Binnenthaler Eisenglanze, angenommen, dass das auftre!ende primäre Rhomboëder stets das positive sei, und wurden demgemäss die Vorzeichen der Skalenoëder und Rhomboëder bestimmt. Sollte sich auf irgend eine Weise, etwa durch Aetzversuche, herausstellen, dass an einigen Typen die als das positive primäre Rhomboëder gedeuteten Flächen dem negativen primären Rhomboëder zugehören, so müssten dem entsprechend die Vorzeichen auch der übrigen an jenen Krystallen vorhandenen Formen geündert werden.

Die vorliegenden Untersuchungen haben zur Auffindung von 01 weiteren am Eisenglanz und Titaneisen vom Binnenthal noch nicht beobachtelen Flächen geführt, von welchen nur 3 bereits von anderen Vorkommnissen bekannt sind. Die früher (Bd. I, S. 581) gegebene erste Tábelle erhält daher folgenden Zuwachs :

$$
\text { 10. } u=\frac{1}{4} R(10 \overline{4} 4) ; 11 . \varphi=\frac{5}{8} R(50 \overline{5} 8) ; 12 . N=-\frac{5}{4} R(05 \overline{5} 4) \text {. }
$$

Die andern 7 sicher bestimmten Formen sind neu für den Eisenglanz überhaupt; sie sind der zweiten Tabelle in folgender Weise anzureihen :

\begin{tabular}{|c|c|c|c|c|c|c|c|c|c|c|}
\hline \multirow{3}{*}{$\begin{array}{l}\text { 18. } \quad(9.5 . \overline{4} \overline{4} .6) \\
\text { 19. } \Pi=(50 \overline{5} 4)\end{array}$} & \multirow{3}{*}{$\begin{array}{c}\infty R \frac{7}{2} \\
\frac{5}{4} R\end{array}$} & \multirow{3}{*}{$\begin{array}{c}\infty_{\frac{5}{4} P}^{\frac{14}{9}} \\
\frac{2 P}{2}\end{array}$} & \multicolumn{2}{|c|}{$\mathrm{U}$} & \multicolumn{2}{|c|}{$\mathrm{X}$} & \multicolumn{2}{|c|}{$\mathrm{Y}$} & \multicolumn{2}{|c|}{ Z } \\
\hline & & & $90^{0}$ & $0^{\prime}$ & & 16 & 180 & $44^{\prime}$ & & $0^{\prime}$ \\
\hline & & & 63 & 0 & 101 & & 101 & 0 & 79 & 0 \\
\hline 20. $\Theta=(20 \overline{2} 1)$ & $2 R$ & $\frac{2 P}{2}$ & 7.2 & 20 & 111 & 13 & 111 & 13 & 68 & 47 \\
\hline$(05 \overline{5} y)$ & $-\frac{5}{3} R$ & $-\frac{\frac{5}{9} P}{z}$ & 41 & 6 & 69 & 24 & 69 & 24 & 110 & 36 \\
\hline$(0,8 . \overline{8}, 11)$ & $-\frac{8}{11} R$ & $-\frac{\frac{8}{11} P}{2}$ & 48 & 48 & 77 & 56 & 77 & $\ddot{3} 6$ & 102 & 4 \\
\hline 23. $\quad J=x(5.9 . \overline{4} \overline{4} \cdot 20)$ & $-\frac{1}{5} R \frac{1}{2}$ & $-\frac{{ }^{\frac{1}{0}} P P}{z}$ & 43 & 59 & 52 & 16 & 28 & 20 & 93 & 34 \\
\hline 24. $\Sigma=x(1.15 . \overline{6} \cdot 4)$ & $-\frac{7}{2} R \frac{6}{7}$ & $-\frac{4 P \frac{16}{15}}{2}$ & 80 & 41 & 111 & 20 & 6 & 20 & & 31 \\
\hline
\end{tabular}


Was den ehedem von mir beschriebenen Titaneisenkrystall betrifft, so will ich noch erwähnen, dass Herr Sadebeck*) die Ausbildung dieses Krystalls (vgl. Fig. 7, Taf. XXIV. Bd. I dieser Zeitschr.) dadurch erklären will, dass er fur die beiden in der hemiëdrischen Ausbildung zusammenfallenden Flächen des Hexagondodekaëders $n$ eine verschiedene Beschaffenheit annimmt, während ich, insbesondere wegen der eigenthümlichen Entwicklung der Flächen $q, T, n, n^{\prime}$ und $R$ den Krystall für tetartoëdrisch ausgebildet halte und als Zwilling nach der Basis betrachte, ohne jedoch die Ausbildung dieses Krystalls als Beweis fur die Tetartoëdrie des Titaneisens überhaupt gelten zu lassen. Ich muss es dem Leser tuberlassen, nach genauerem Studium meiner Beschreibung und der erwähnten Figur sich für die eine oder andere Ansicht zu entscheiden. Nur will ich, um Missverständnissen vorzubeugen, bemerken, dass die Figur zum Theil eine ideelle ist, indem das von Herrn Sad ebeck nicht aufgefundene Rhomboëder $Z$, für welches ich den Winkel zur Basis gemessen habe, nicht vollflächig auftritt, und ferner, dass die von Herrn Sad ebeck fur $T$ angenommene Zone, wie ich mich ehedem durch Messung überzeugt habe, nicht existirt, also für diese Fläche mein Zeichen beizubehalten -ist.

Schliesslich möchte ich noch auf einige in meiner früheren Arbeit (diese Zeitschr. Bd. I, p. 562-582) stehen gebliebene Druckfehler aufmerksam machen, die ich zu berichtigen bitte. Es ist zu setzen :

S. 562 . Z. 4 v. U.: "rhomboëdrisch-pyramidal" statt wrhomboëdrisch, pyramidal«.

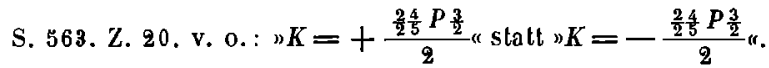

S. 564 . Z. 9 v. 0.: "- $\frac{1}{2} \frac{0}{7} R 3$ « statt $" \frac{1}{2} \frac{0}{7} R 3$ \%.

S. 567. Z. 15 v. o.: nesi slall "Dic.

" "Z. 19 v. 0.: „den Skalenoëdern« statt »die Skalenoëder

" Z. 15 v. u. : $" R(10 \bar{T} 1)$ « stalt $n(0001) \%$.

S. 568. Z. 6 v. o.: $" 553^{0} 48^{\prime}$ ' statt $149048^{\prime}$ '.

S. 569. Z. 3 v. u. : $1265^{0} 44^{\prime} \ll$ slatt $\gg 65^{0} 39^{\prime} \ll$.

" Z. 1 u. 2 v. u. : "mit der darunter liegenden Fläche $F$ einschliesst, also den Winkel (7.14. $\overline{2} \bar{T} .18,5.9 . \overline{1} \overline{4} . \bar{T} \overline{3} ; \ll$ statt " mit der anliegenden Fläche $F \ldots$ (7. 14. $\overline{21} .18,9.5 . \overline{1} \overline{4} .13) \propto$.

S. 570. Z. 4 พ. 0.: "Sie zeigen" statt "Er zeigk".

S. 574. Z. 8 v. o.: " - $\frac{\frac{8}{7} P_{2}^{4} \frac{9}{5}}{2}$ “ statt "- $\frac{\frac{6}{7} P_{2}^{4} \frac{9}{6}}{2}$ ".

S. $581 . Z$. 13 v. o.: $\geqslant 0 R$ " statt $" c R$ «.

" Z. 5 v. u. : $\nu \nu$ a statt " $r$ ".

S. 582. Z. 6 v. u. : $" 53^{0} 48^{\prime}$ 's statt $149^{\circ} 48^{\prime}$ ' und $284^{\circ} 28^{\prime}$ ' slatt $" 62^{\circ} 32^{\prime}$ «.

*) N. Jahrbuch f. Min. 1878. p. 287 u. f. 